\title{
Usher Syndrome Type 2C
}

National Cancer Institute

\section{Source}

National Cancer Institute. Usher Syndrome Type 2C. NCI Thesaurus. Code C153174.

An autosomal recessive sub-type of Usher syndrome caused by homozygous or

compound heterozygous mutation(s) in the ADGRV1 gene, encoding adhesion G protein-

coupled receptor V1. It may also result from biallelic digenic mutation(s) in ADGRV1 and

PDZD7, which encodes PDZ domain-containing protein 7. 